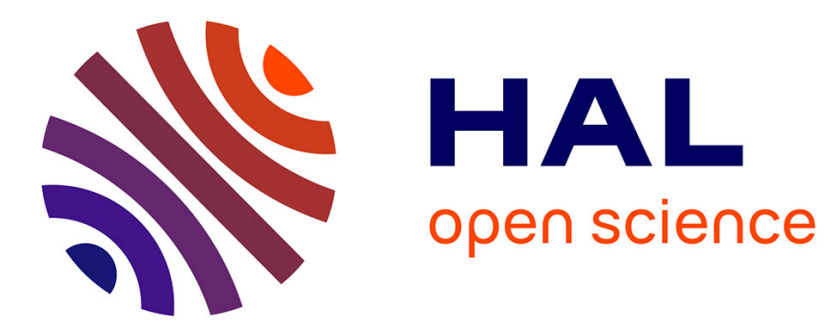

\title{
Some comments on scaling exponents of turbulence
}

\author{
Christian Baudet, S. Ciliberto, Phan Tien
}

\section{To cite this version:}

Christian Baudet, S. Ciliberto, Phan Tien. Some comments on scaling exponents of turbulence. Journal de Physique II, 1993, 3 (3), pp.293-299. 10.1051/jp2:1993133 . jpa-00247833

\section{HAL Id: jpa-00247833 https://hal.science/jpa-00247833}

Submitted on 1 Jan 1993

HAL is a multi-disciplinary open access archive for the deposit and dissemination of scientific research documents, whether they are published or not. The documents may come from teaching and research institutions in France or abroad, or from public or private research centers.
L'archive ouverte pluridisciplinaire HAL, est destinée au dépôt et à la diffusion de documents scientifiques de niveau recherche, publiés ou non, émanant des établissements d'enseignement et de recherche français ou étrangers, des laboratoires publics ou privés. 
Classification

Physics Abstracts

47.25

\title{
Short Communication
}

\section{Some comments on scaling exponents of turbulence}

\author{
C. Baudet, S. Ciliberto and Phan Nhan Tien \\ Ecole Normale Supérieure de Lyon, 46 Allée d'Italie, 69364 Lyon Cedex 07, France
}

(Received 23 November 1992, accepted 22 December 1992)

\begin{abstract}
Résumé . - Quelques auteurs ont prétendu observer dans les écoulements turbulents un accroissement de l'exposant de la loi de puissance pour la fonction de structure du premier ordre de la vitesse lorsque le nombre de Reynolds décroît. D'importantes déductions relatives à la transition vers la turbulence pourraient être tirées de ce résultat. Cependant, il ressort des résultats expérimentaux que nous avons obtenus récemment que ce résultat ne provient que d'une définition incorrecte du domaine inertiel. Nos données expérimentales prouvent clairement que les exposants des fonctions de structure de la vitesse ne varient pas avec le nombre de Reynolds, ce qui est cohérent avec le caractère auto-similaire des fluctuations de vitesse en turbulence.
\end{abstract}

\begin{abstract}
Several authors have reported that in turbulence the scaling exponent of the first order velocity structure function increases when the Reynolds number Re decreases. From this result some important consequences on the transition to turbulence could be obtained. However we report experimental evidence that this result is coming only from an improper definition of the inertial range. Our data clearly show that the scaling exponents remain constant as a function of Re which is consistent with the selfsimilarity of spectra.
\end{abstract}

One of the signatures of fully developed turbulence is the existence of power laws in spectra and in structure functions (see below) of the variables of turbulent flows [1]. An important issue in the study of turbulence is how the exponents of these power laws behave as a function of the control parameter, namely the Reynolds number $\operatorname{Re}=U L / \nu$, where $U$ is the mean velocity, $L$ a characteristic length and $\nu$ the kinematic viscosity. Specifically one would like to understand whether these exponents either change or remain constant when Re approaches the value $R c$, where the transition to turbulence is observed. Indeed some theories [2] and experiments [3, 4] suggest a self similar behaviour of spectra recorded at different $R e$, as a consequence the exponents have to be constant. Vice versa some measurements on grid turbulence [5] seem to indicate that when $\mathrm{Re} \rightarrow \mathrm{Rc}$ from above the exponents increase and this could be interpreted using the $\beta$ model $[6]$ as the evidence that the dimension $D$, of the space where the dissipation occurs, changes from almost 3 at high Re to 2 at Re close to $R c \simeq 300$. A theoretical paper 
[7] tried to explain this result which is indeed very appealing because it permits to clearly identify $R c$ as the transition point to fully developed turbulence and eventually to trace an analogy to second order phase transition, where $D$ would be an order parameter. In order to understand these results, we have done a series of measurements in our wind tunnel. We find that the result of reference [5], which has also been quoted in several papers [8,9], is coming from the fact that the experimental data have not been properly analysed and interpreted. Thus the purpose of this paper is to make these points clear to avoid other misinterpretation of experimental results and other theoretical efforts to explain them.

Before discussing these results we need to briefly remind the $\beta$ model predictions [6], which are used in reference [5]. In order to characterize the turbulent flow experimentally, one can measure, for example, one velocity component $V$ and construct the velocity difference $\Delta V=$ $[V(x+r)-V(x)]$ between two points at a distance $r$. Then one can compute the velocity structure function $S_{n}(r)=\left\langle|\Delta V(r)|^{n}\right\rangle$ where $\langle$.$\rangle stands for ensemble average. The S_{n}(r)$ should scale scale as a function of $r$ in the following way:

$$
S_{n} \propto r^{S_{n}}
$$

with $r$ in the inertial range which is the range where viscosity effects are negligible. The $\beta$ model [6] predicts that, for $\operatorname{Re} \rightarrow \infty$,

$$
\zeta_{n}=n \cdot h+3-D
$$

with $h=(D-2) / 3$, where $D$ is the fractal dimension of the space where the dissipation occurs. At high $\operatorname{Re}, D$ is about 2.9. Notice that if $D=3$ then $h=1 / 3$ that is the Kolmogorov value. Furthermore for $n=3$ one recovers $\zeta_{3}=1$, independently of $D$, as it must be because of the Kolmogorov exact relation

$$
\left\langle\Delta V^{3}\right\rangle \propto r
$$

with $r$ in the inertial range [10]. More correctly, instead of the $\beta$ model one could use the multifractal formalism [11-13] but for low order moments the fractal and multifractal models are not distinguishable.

We now discuss the results of reference [5]. In this paper the authors describe an experiment where a heterodyne technique allows them to measure velocity differences. With this technique they characterized a grid turbulent flow as a function of Re, in terms of the first order structure function. Decreasing Re from 2000 to 200 they found that $\zeta_{1}$ increases from about 0.4 to 1 . They interpreted the result $\zeta_{1} \simeq 1$ for $\mathrm{Re} \leq \mathrm{Rc}=300$ in terms of the $\beta$ model by extending it at small Re. As, from equation $2, \zeta_{1}=(7-2 \cdot D) / 3$, they conclude that if $\zeta_{1} \simeq 1$ then $D=2$ and $h=0$. This result could have several consequences. First of all the extension of the $\beta$ model at small $\operatorname{Re} \leq \mathrm{Rc}$. Secondly $D$ changes from $D \simeq 2.9$ at high $\operatorname{Re}$ to $D=2$ for $\operatorname{Re} \leq \mathrm{Rc}$. Thirdly it has been recently shown [14] that $h$ is equal 0 only for shock waves, thus the above mentioned interpretation of the experimental data would imply that these waves can occur in turbulent flows at small Re. However from equation (2) one sees that $h=0$ implies $\zeta_{n}=1$ for all $n$, but such a simple test has not been done to enforce this claim.

Thus to give more insight into these questions we have performed a series of measurements in our wind tunnel. Our experimental set-up is a standard one. A small wind tunnel with a square cross-section of $50 \mathrm{~cm}$, is used to produce an air flow with a mean speed which can be adjusted between 0.2 and $10 \mathrm{~m} / \mathrm{s}$ with a residual level of turbulence at the maximum speed of about $0.3 \%$. Turbulence has been produced either with a grid or with a cylinder. The two dimensional grid is constructed with aluminium rods of $2.5 \mathrm{~cm}$ diameter with their axis $5 \mathrm{~cm}$ apart. The cylinder has a diameter of either 5 or $10 \mathrm{~cm}$ in order to change Re without 
changing the speed. In the case both of the grid and of the cylinder we made measurements at two different down stream locations in order to verify the stability of the results as a function of the distance. One of the two locations was at 10 times and the other at 20 times the integral length $L$, that is to say, the grid spacing and the cylinder diameter respectively. With this setup, the Reynolds number based on the Taylor scale $R_{\lambda}$ is in the range $100-500$, corresponding to $\operatorname{Re}$ based on the integral scale of $2000-40000$. The maximum turbulence level was between 7 and $15 \%$. The velocity measurements were done using a TSI hot film detector of sensing length $1 \mathrm{~mm}$ and diameter $50 \mu \mathrm{m}$. The hot film was controlled by TSI IFA100 constant temperature hot wire anemometer. The velocity signal was digitized with a 16 bits A/D converter at a rate of $10 \mathrm{kHz}$ which is sufficiently high in order to avoid aliasing problems in the range of Re studied. At least $10^{6}$ data points have been recorded for each value of $R e$ in order to have enough statistical accuracy. All the data have been corrected to take into account the nonlinear response of the hot film and the Taylor hypothesis has been used to obtain the spatial dependence of $V$, i.e. $r=U \cdot t$ where $U$ is the mean velocity of the wind.
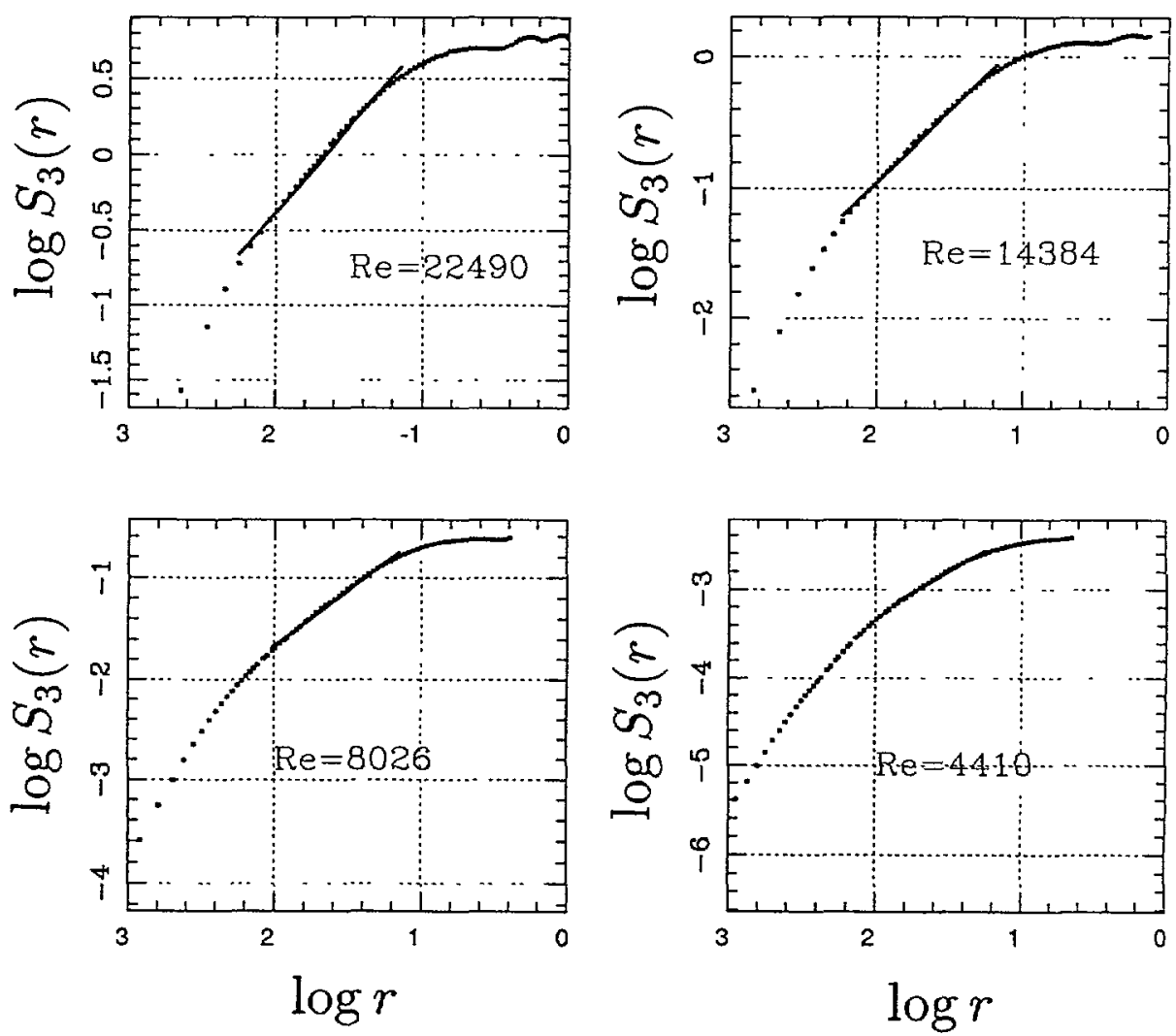

Fig. 1. $-S_{3}$, for the case of turbulence behind a cylinder, computed at four different $R e=22490$; $14384 ; 8026 ; 4410$ corresponding to $R_{\lambda}=350 ; 281 ; 210 ; 156$ respectively. The solid straight lines of slope 1 indicate the region where this linear fit is possible with a $5 \%$ accuracy. 


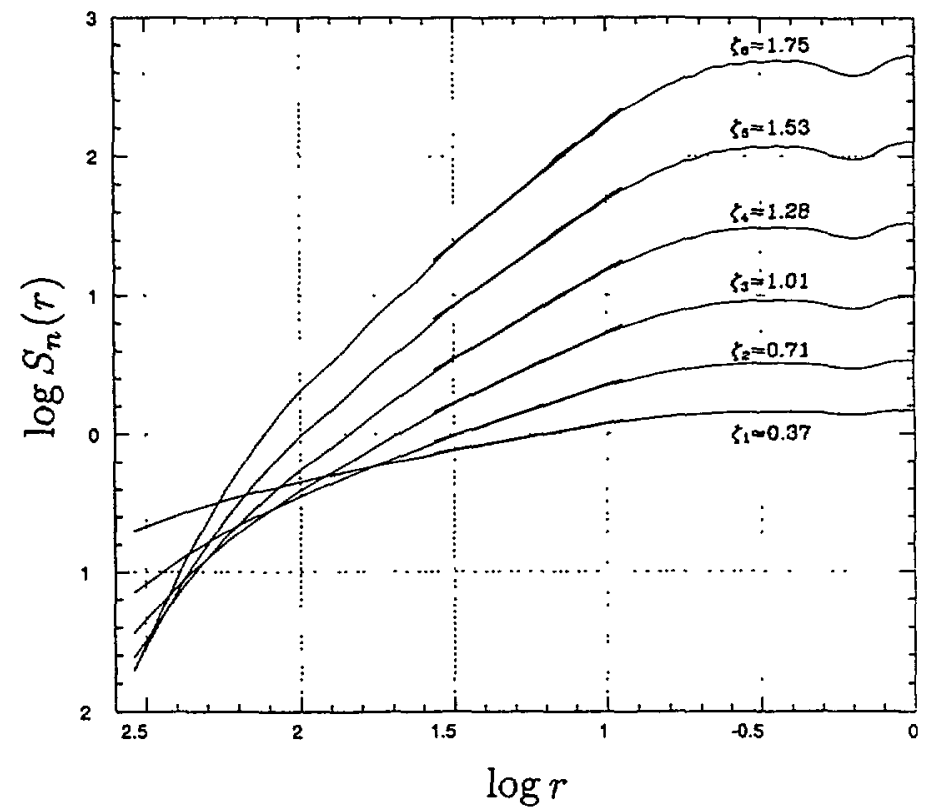

Fig. 2. $-S_{n}$ for different $n$ as a function of $r$ at $R e=26000, R_{\lambda}=378$. Solid lines indicate the regions where the fits have been done.

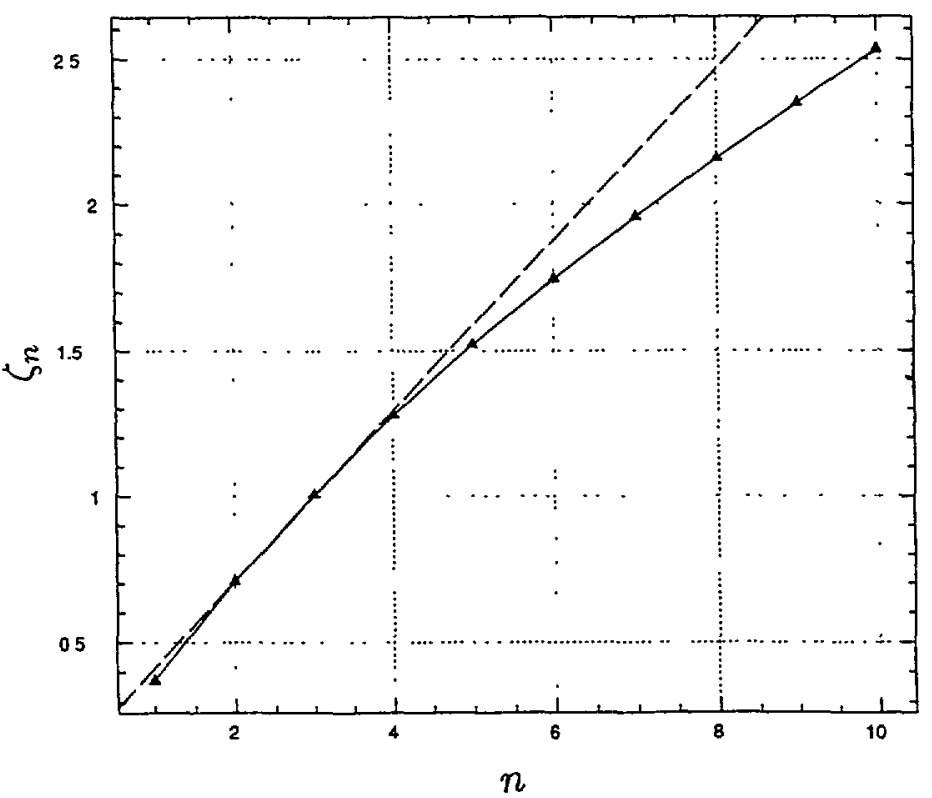

Fig. 3. $-\zeta_{n}$ versus $n$ at $\operatorname{Re}=26000$. The dashed line is the $\beta$ model prediction. The error of $\zeta_{n}$ is less than $5 \%$. 


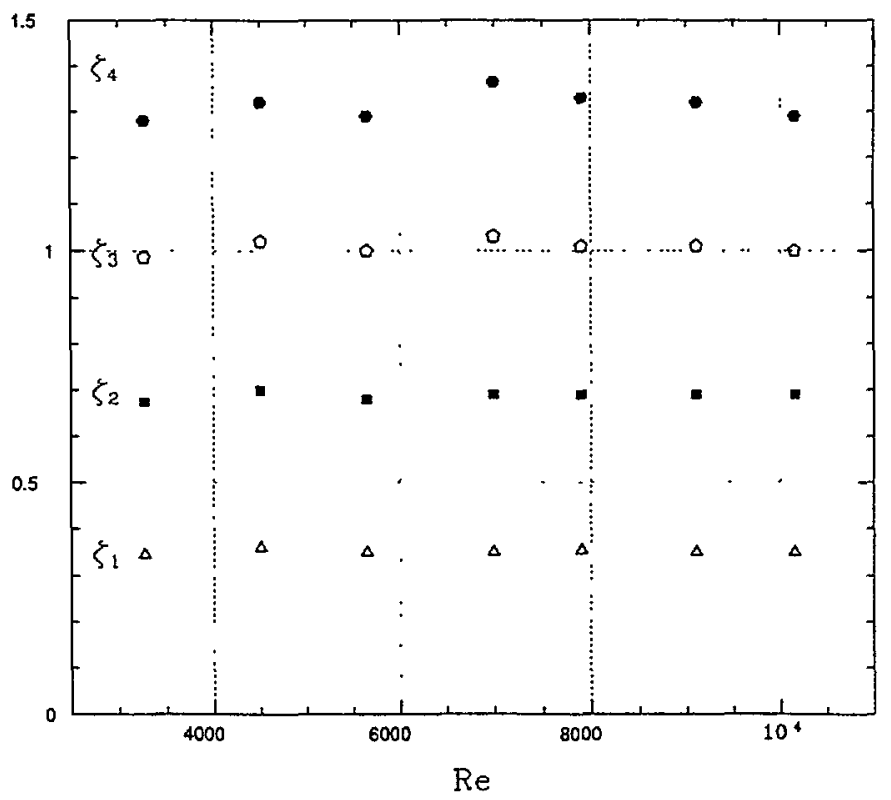

Fig. 4. $-\zeta_{n}$ for $n=1,2,3,4$ as a function of Re.

Here we describe only the results obtained with the cylinder but the grid gives exactly the same results. The data have been analysed in the following way. We have first computed $S_{3}(r)$ and the results are reported in figure 1 for different Reynolds numbers. For each value of Re we identify the inertial range by using the Kolmogorov exact relation, (Eq. (3)), specifically we found the interval in $r$ where $\frac{\log S_{3}(r)}{\log r}$ is constant [15]. The solid straight lines of slope 1 in figure 1 show the regions in $r$ where this fit is possible, with a $5 \%$ accuracy. We see that the length of the interval becomes shorter and shorter when Re is decreased. We find that the length of the so defined inertial range scaled correctly as $\mathrm{Re}^{0.75 \pm 0.1}$ which is consistent with the Kolmogorov law $\mathrm{Re}^{3 / 4}$

The determined inertial range is then used to make a best fit of the other moments $S_{n}(r)$ in order to evaluate $\zeta_{n}$ for several $n$. An example is shown in figure 2 where the different $S_{n}(r)$ measured at $\operatorname{Re}=26000\left(R_{\lambda}=378\right)$ are shown as a function of $r$. The solid straight lines indicate the regions where the fits have been done. The measured $\zeta_{n}$, reported in figure 3 versus $n$ are in agreement with previous measurements [15] made at Re much higher than those reachable in our experiment.

We have repeated the same operations to determine the $\zeta_{n}$ at lower Re. As we notice in figure 1, by lowering Re the inertial range (solid lines in Fig. 1), shrinks but we find that the value of $\zeta_{n}$, for all $n$, measured in this region do not change with Re. This is shown in figure 4 where the measured value of $\zeta_{n}$ for $n=1,2,3,4$ are plotted as a function of Re. We clearly see that no noticeable change is observed in the different exponents measured at different Re.

The question is now to understand where the result of reference [5] is coming from. The answer is quite simple, because this result is due to the fact that the inertial range was not properly defined. Indeed in reference [5] the interval, where the best fit of the structure function $S_{1}(R)$ was done, was kept fixed as a function of Re. In order to verify our interpretation, we 


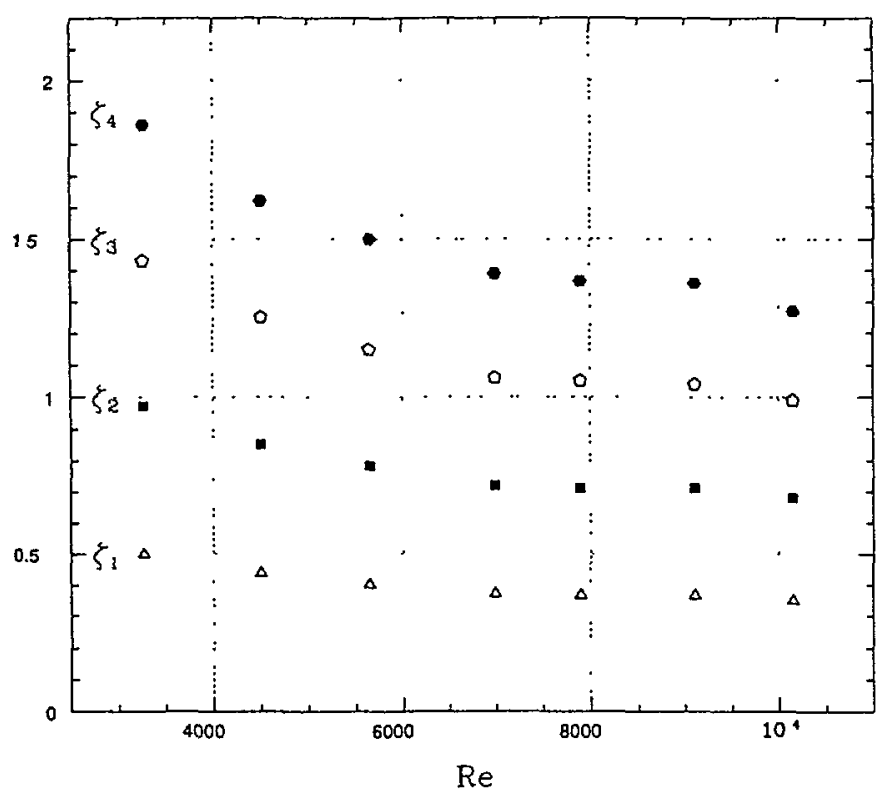

Fig. 5. $-\zeta_{n}$ for $n=1,2,3,4$ as a function of Re using the same data already used to obtain the results shown in figure 4 but performing the analysis as in reference [5].

have analysed our data by keeping constant the range in which the fits of structure functions were done. Specifically this range was equal to the inertial range obtained for the maximum Re. The results obtained by using this wrong definition of inertial range are shown in figure 5 where the values of $\zeta_{n}$ for $n=1,2,3,4$ are shown as a function of Re. The result of reference [5] is now recovered. We clearly see that $\zeta_{1}$ increases when Re decreases and reaches the value 0.5 for the smallest $R e$ available. Here we stopped the analysis at the same Re of figure 4 because this is the minimum $R e$ in which an inertial range can still be observed. However extending this analysis at smaller $\operatorname{Re}$ we find that $\zeta_{1}$ increases further. From the result of figure 5 we also understand that the interpretation of reference [5], based only on $\zeta_{1}=1$, is not correct. Indeed by performing the same analysis of reference [5] on all structure functions we see that all the $\zeta_{n}$ increase making the claim $h=0$ absolutely inconsistent with experimental results.

As a conclusion we have shown using experimental results of turbulence behind a grid and a cylinder that the exponents $\zeta_{n}$ do not change as a function of Re but they remain constant which is indeed in agreement with the selfsimilarity of spectra [2-4] and extended self similarity [16]. As a consequence all theoretical interpretations based on the increasing of the scaling exponents have no experimental justification. We want to stress that this result has nothing to say about the transition, from to 2.8 to 2 , observed in the dimension $D^{\prime}$ of isoscalar surfaces [9]. What is certainly true is that $h$ and $D^{\prime}$ are not simply related as proposed in references $[8,9]$, because $h(\mathrm{Re})$ is constant whereas $D^{\prime}$ is a function of Re.

Furthermore when this paper was in preparation we became aware of a preprint [17] where, using the same technique of reference [5], the authors claim that $D$ remain constant as a function of Re which agrees with our results and is in contrast with their previous measurements. 


\section{Acknowledgements.}

S.C. acknowledges useful discussions with W. Goldburg.

\section{References}

[1] Kolmogorov A.N., Dokl. Akad. Nauk. SSR 30 (1941) 301.

[2] Frisch U., Vergassola M., Europhys. Lett. 14 (1991) 439.

[3] Gagne Y., Castaing B., C. R. Acad. Sci. Paris Serie II 312 (1991).

[4] Wu X.Z., Kadanoff L., Libchaber A., Sano M., Phys. Rev. Lett. 64 (1990) 2140.

[5] Tong P., Goldburg W.I., Chan C.K., Sirivat A., Phys. Rev. A 37 (1988) 2125.

[6] Frisch U., Sulem P., Nelkin M., J. Fluid. Mech. 87 (1974) 719.

[7] Huber G., Alstrom P., J. Phys. A: Math. Gen. 24 (1991) L1105.

[8] Costantin P., Procaccia I., Sreenivasan K.R., Phys. Rev. Lett. 67 (1991) 1739.

[9] Sreenivas an K.R., Ann. Rev. Fluid Mech, 23 (1991) 539.

[10] Monin A.S., Yaglom A.M., (MIT Press, Cambridge, 1975).

[11] FrisCh U., PARISI G., Proceedings of the International School of Physics E. Fermi, 1983, Varenna Italy, M. Ghil, R. Benzi, G. Parisi Eds. (1985).

[12] Benzi R., Paladin G., Parisi G., Vulpiani A., J. Phys. A: Math. Gen. 17 (1984) 3521.

[13] Meneveau C., Sreenivasan K.R., Nuclear Phys. B2 (1987) 49.

[14] Frisch U., Proceedings of the Les Houches School, Turbulence in spatially extended systems, R. Benzi, S.S. Ciliberto, C. Basdevant Eds. (1992).

[15] Anselmet F., Gagne Y., Hopfinger E.J., Antonia R.A., J. Fluid. Mech. 140 (1984) 63.

[16] Benzi R., Ciliberto S., Tripiccione R., Baudet C., Massaioli F., Succi S., submitted for publication.

[17] PAK H.K., GoldBURG W.I., preprint 1992. 